Cahiers de recherches médiévales

Figures mythiques médiévales auX $\mathrm{XIX}^{\mathrm{e}}$ et $\mathrm{XX}$ siècles

\title{
Fées, sorciers, princesses
}

Figures mythiques médiévales dans la poésie de Jean Lorrain

\section{Liana Nissim}

\section{CpenEdition}

\section{Journals}

Édition électronique

URL : https://journals.openedition.org/crm/1823

DOI : $10.4000 / \mathrm{crm} .1823$

ISSN : 1955-2424

Éditeur

Honoré Champion

\section{Édition imprimée}

Date de publication : 15 décembre 2004

Pagination : 165-180

ISSN : $1272-9752$

Référence électronique

Liana Nissim, «Fées, sorciers, princesses », Cahiers de recherches médiévales [En ligne], 11 | 2004, mis en ligne le 10 octobre 2007, consulté le 15 décembre 2022. URL : http://journals.openedition.org/crm/ 1823 ; DOl : https://doi.org/10.4000/crm.1823

Ce document a été généré automatiquement le 15 décembre 2022.

Tous droits réservés 


\title{
Fées, sorciers, princesses
}

\author{
Figures mythiques médiévales dans la poésie de Jean Lorrain
}

\section{Liana Nissim}

\begin{abstract}
À n'en pas douter, ce fut une singulière époque que ce Moyen Âge [...]. Pour les uns, il est entièrement blanc et pour les autres, absolument noir ; aucune nuance intermédiaire ; époque

d'ignorance et de ténèbres, rabâchent les normaliens et les athées ; époque douloureuse et exquise, attestent les savants religieux et les
\end{abstract} artistes.

(J.-K. Huysmans, Là-bas)

1 S'il faut en croire les paroles de Huysmans citées en exergue, selon qui le Moyen Âge est une époque douloureuse et exquise pour les savants religieux et les artistes, nous sommes contraints d'accueillir Jean Lorrain parmi les artistes, étant donné qu'on ne peut d'aucune manière lui attribuer un savoir religieux quelconque, et du moment qu'un Moyen Âge effectivement exquis et douloureux parcourt tout un pan de sa vaste et très diverse production.

2 J'ai déjà eu l'occasion de souligner que Jean Lorrain - dans les limites certes d'un maniérisme absolu, jamais dépassé - est un artiste rien moins que méprisable ; et il est assez difficile de comprendre les critiques qui, même s'ils consacrent des études ou des livres entiers à cet auteur, se croient obligés d'exhiber à tout moment un détachement méprisant ou une cinglante ironie à ses égards ${ }^{1}$.

Incontestablement, Jean Lorrain a tout fait pour noircir son personnage, pour s'attribuer une très mauvaise réputation, pour s'afficher comme le héraut du vice et de la fange, «l'ambassadeur de Sodome à Paris » $^{2}$ de la sorte, « ce fanfaron du vice [...] se retrouve peu à peu prisonnier des apparences et de sa légende. [...] [son] exagération [...] devient l'arme que ses détracteurs retourneront si facilement contre lui. [...] Sa réputation entachera sa littérature $»^{3}$. Heureusement, les études plus récentes sur la fin de siècle, et plus particulièrement celles de Jean de Palacio et de Guy Ducrey, offrent de Jean Lorrain et de son œuvre un tableau beaucoup plus équilibré, libre enfin des 
préjugés qui l'avait frappé dès son vivant. Relisons, à titre d'exemple, le portrait très équitable que donne de lui Guy Ducrey :

Jean Lorrain (1855-1906) est l'une des figures les plus intéressantes du Paris fin-desiècle. Poète, romancier, homme de théâtre, il était surtout célèbre à l'époque pour son travail de journaliste et de chroniqueur. D'un trait de plume, il faisait et défaisait les réputations, assurait le succès d'une pièce et la faillite d'une autre, défendait des talents encore inconnus, pourfendait les imposteurs, toujours partisan, toujours redoutable et bouillonnant. Il affichait dans sa vie des attitudes d'esthète homosexuel en un temps où elles étaient une provocation, et ses afféteries, ses collections de bibelots, ses bagues donnaient lieu à de fréquentes caricatures. Ce grand dénonciateur des vices contemporains semblait ainsi incarner la décadence en sa personne et, de mots d'esprit en phrases assassines, promenait partout sa mélancolie et son pessimisme comme une fleur violette à sa boutonnière. 4

4 Or, parmi les préjugés que la critique n’a manqué d'entretenir constamment on retrouve souvent un très dur jugement sur ses œuvres poétiques : «la poésie le sert mal. Pour nous l'horreur tourne vite au comique » écrit par exemple Philippe Jullian ${ }^{5}$; et encore : « son œuvre, en partie déplorable, n'a échappé à aucun des mauvais goûts de son temps [...]. Cet homme, qui s'est cru poète, était avant tout un merveilleux journaliste $»^{6}$.

5 Loin de moi l'idée de magnifier la production poétique de Jean Lorrain comme une suite de chefs-d'œuvre ; j'insiste au contraire sur son maniérisme extrême, peu favorable ou peu capable d'innovation; mais il s'agit d'un maniérisme souvent séduisant, très raffiné, à même d'aboutir fréquemment à un chiffre bien personnel et d'exprimer dans son ensemble une vision du monde suggestive et cohérente, une poétique qui est en même temps une solide barrière opposée à la réalité tant exécrée et une voie ouverte sur un rêve aussi fragile qu'artificiel, constamment marqué par les connotations les plus voyantes de la littérature fin de siècle.

6 Bien évidemment, dans une œuvre poétique de ce genre, maniériste et exemplaire en même temps, l'imagerie moyenâgeuse ne pouvait pas ne pas occuper une place remarquable.

7 Comme le rappelle Anna Maria Finoli, le thème du Moyen Âge s'impose au XIX ${ }^{e}$, dès son commencement, en tant que

tema già preromantico - genre troubadour, ruinismo, gusto per la poesia "popolare" -, ma certo squisitamente romantico per le connotazioni che il concetto, e diremmo meglio il mito, del Medio Evo ha assunto nel tempo e che il romanticismo ha fatto proprie. ${ }^{7}$

Cependant, s'il est vrai que pour les écrivains romantiques «la figura dell'uomo medievale aderisce a quella dell'eroe romantico, con una netta prevalenza del côté eroico $\aleph^{8}$, les choses sont un peu différentes dans la seconde moitié du siècle, surtout après l'œuvre de Baudelaire et de Wagner: le Moyen Âge reste, bien sûr, une « età tenebrosa, barbarica, "gotica" $\gg$, une source de suggestions infinies, un catalogue inépuisable d'images; mais l'ambiance générale se transforme; les références historiques s'estompent en faveur de la légende et du merveilleux, les personnages perdent leur caractère haut en couleurs, violent et passionné, pour s'effiler dans une rêveuse exténuation ; une atmosphère plus floue et brumeuse se diffuse, crépusculaire, mystérieuse et volontiers mystique, comme si l'ensemble des images supposées médiévales pouvaient être le haut lieu d'une anti-réalité, ou plutôt d'une irréalité souhaitée pour la révélation de tous les possibles, pour la quête idéale de l'absolu. Tel 
est, dans ses grandes lignes, le Moyen Âge mis en place par Jean Lorrain dans ses poèmes. En voici une suite de courts exemples :

Dans l'ombre et le secret d'un manoir à sept tours,

Aux sons d'une sirvente et d'une mandoline

Que j'aimerais, à l'heure où le soleil décline,

Endormir une reine aux grands yeux de velours ! 10

Les doux propos d'amour et les heures pensives

Le soir, dans la tour ronde aux gothiques ogives,

Qui donne sur les bois, et le lit nuptial

Dans l'ombre et le secret du bourg seigneurial. [...]

Et dans l'air étoilé, le cou tendu, hagarde,

Le guivre de granit est là, montant sa garde

Éternelle et farouche aux créneaux du manoir. ${ }^{11}$

Et l'homme à feutre gris sourit. Sa mine lasse,

Sa pâleur, sa main longue et l'exquis nonchaloir

De sa pose et ses yeux ivres de désespoir

Contaient tout un hautain passé de grande race :

Une enfance princière au fond d'un vieux manoir

Entre des lévriers et des chevaux de chasse

Et de longs entretiens les soirs à la terrasse

Avec des cavaliers masqués de velours noir. ${ }^{12}$

9 On peut le constater déjà à travers ces quelques vers: châteaux, forêts, reines et chevaliers s'esquissent pour composer un médiévalisme éloigné désormais de toute référence au domaine de l'histoire, plongé entièrement dans une vague atmosphère entre la légende et le conte de fées, des genres pour lesquels Jean Lorrain n'a jamais cessé de nourrir un engouement profond, comme il le reconnaît lui-même dans la préface à ses Princesses d'ivoire et d'ivresse :

Ces contes de fées, qu'on a remplacés aujourd'hui par des livres de voyages et de découvertes scientifiques, ces merveilleuses histoires qui parlaient au cœur à travers l'imagination et préparaient à la pitié par d'ingénieux motifs de compassion pour de chimériques princesses, dans quel ravissement de petite âme éblouie et frémissante ont-elles bercé les premières années de ma vie ! [...]

Il faut aimer les contes, il faut s'en nourrir et s'en griser [...].

Pour moi, je l'avoue, je les ai adorés et d'une adoration presque sauvage. ${ }^{13}$

Aussi, dans ce Moyen Âge féerique et légendaire tout le peuple magique des contes surgit, de merveilleuses princesses éblouies, de mélancoliques princes charmants, des fées mystérieuses et redoutables, des lutins, des sorciers, des forêts enchantées... : Jean Lorrain, ainsi que son je poétique, est assurément «un envoûté des gothiques magies $»^{14}$.

11 Reines, princesses, nobles jeunes filles défilent nombreuses dans les vers de Lorrain; de l'une d'elles il raconte toute la légende dans un long poème composé de cinq sonnets, dont le premier trace le portrait de la jeune fille :

C'était une pensive et douce créature

Aux épaules frêles, froides, comme azurées,

Aux petites oreilles jamais effleurées

D'aveux d'amour.

Un parc à l'ondoyant murmure

La gardait dans son ombre invisible et murée.

Parmi la clématite et la pourpre des mûres

Elle errait, blanche et calme, écartant les ramures,

Et les lilas neigeaient sur sa tête dorée. 
Son père, un vieux baron, guerroyait dans de vagues

Et très lointains pays pour un roi de Bohême,

Et l'enfant solitaire, assise entre les vagues

De verdure, épelait quelqu'antique poème

Ou suspendait distraite une à une ses bagues

Aux tiges des roseaux empanachés d'or blême. ${ }^{15}$

12 Dans les sonnets suivants, on découvre que la belle fille, si seule et prisonnière dans son vaste domaine, est fatalement attirée par la lourde et magnifique couronne parsemée de pierres précieuses resplendissantes, posée dans un coffret d'opale; elle adore se parer de cette couronne, même si, chaque fois qu'elle l'essaye, elle tremble de peur,

Un vieux conte enfantin voulant, sombre ironie,

Que le nimbe étoilé, qui rayonne et fleuronne

Aux tempes du baron, face dure et jaunie,

Se brisât au front blanc de la jeune baronne. ${ }^{16}$

Dans la vaste salle qui garde la couronne, il y a une immense verrière où sont peintes trois horribles et vieilles filandières (repoussant avatar des trois Parques) que la jeune fille, attirée par la couronne resplendissante, n'a jamais vraiment remarquées. Mais un jour où elle s'apprêtait à poser la couronne sur ses cheveux, elle se voit tout à coup cernée par les trois hideuses mégères : elle tombe en criant de peur, la couronne se brise et blesse à mort la jeune fille.

D'autres tendres princesses vouées à une mort prématurée surgissent dans l'œuvre de Lorrain, dont la plus saisissante est la « svelte et pure Elaine »:

L'allée est droite, obscure et pleine de pervenches.

Dans le corsage étroit d'une robe à longs plis

Et, les deux bras chargés des lys qu'elle a cueillis,

La svelte et pure Elaine apparaît dans les branches.

[...]

Elaine aux yeux d'aurore, au rire humide et frais

A sa place marquée au jardin des cyprès ;

Elaine avec les lys sera morte à l'automne.

Elaine est destinée aux éternels regrets ;

Et, présageant l'ennui d'une fin monotone,

Pâle et froide à ses pieds fleurit une anémone. ${ }^{17}$

Mais le poète préfère, à ces jeunes filles si pâles et si frêles, d'autres héroïnes, d'autres reines et princesses, bien plus farouches, belles et cruelles, où revient sous les plus diverses formes, la figure de la femme fatale :

Leurs yeux troublants d'aigue-marine

Ont le languide attrait des flots.

Les lys en feu de leur poitrine

Sèment la guerre et les sanglots.

Leur lèvre est rouge et leurs fronts pâles ;

Et, sous le hennin couleur ciel,

Leurs cheveux roux, semés d'opales,

Bondissent en flots d'hydromel.

Les Héroïnes sont farouches.

Il faut des meurtres et des morts

Pour atteindre au miel de leurs bouches.

Leurs lents baisers sont des remords.

Les batailles, les épopées,

Les trahisons, les vains serments,

Mieux qu'au clair fracas des épées,

Revivent dans leurs noms charmants. 
Mélusine, Yseulte, Genèvre,

Triste comme un appel de cor,

Leur nom baise et meurtrit la lèvre...

Qui l'a dit le redit encor ;

[...] La tunique entr'ouverte aux hanches,

L'or des cheveux en fusion,

Les sveltes reines aux mains blanches

Surgissent, lente vision.

La clarté du songe les baigne,

Allumant en humide éclair

Les perles rondes de leur peigne

Et les tons nacrés de leur chair ;

Et, dans les feuilles trilobées

Des chardons bleus et des lys d'or,

Des reines au temps dérobées

Le clair essaim triomphe encor. ${ }^{18}$

Mélusine, Yseulte, Genèvre : voici quelques noms exemplaires de ces reines-femmes fatales; Lorrain en est si charmé, qu'il choisit d'ouvrir la section "L'ombre d'or » de L'Ombre ardente par une série de sonnets-portraits qui rapprochent le lecteur du mythe proprement dit. Parmi les reines préférées, Mélusine resplendit magnifique et fatale. Déjà elle apparaissait dans La Forêt bleue :

En robe orientale en coiffe sarrasine,

Au parapet jauni la pâle Mélusine

S'accoude et l'avenir est son souci poignant.

Devant l'horizon rouge aux créneaux accoudée,

Elle songe au destin des futurs Lusignan,

Soudain prise à l'aspect de ce grand ciel saignant

D'un vaste et morne ennui des beaux soirs de Judée.

Elle sent, triste et lasse, aux derniers rais du jour

Venir l'heure du charme et des métamorphoses,

Et ses yeux prévenus veulent voir dans les roses

Du couchant, un adieu du monde à son amour.

Déjà grêle et visqueuse au sommet de la tour,

Elle voit ses bras nus verdir sous les écailles

Et le froid du serpent la saisit aux entrailles. ${ }^{19}$

Pourquoi Mélusine est-elle parée d'une robe orientale et d'une coiffe sarrasine? D'où vient sa nostalgie pour la Judée? Fille de la fée Presine et du roi Elinas d'Albanie, arrachée du monde des humains par sa mère, qui a regagné le séjour enchanté d'Avalon, Mélusine n'a avec la Judée qu'un lien bien fragile : l'un de ses fils devient roi de Chypre et en effet Chypre fut "vendue en 1192 à Guy de Lusignan en dédommagement de son éviction du trône de Jérusalem ${ }^{20}$; donc, il faut penser plutôt à une contamination entre la reine fondatrice de la lignée des Lusignan avec d'autres reines, elles aussi chères à Jean Lorrain, Hérodias et sa fille Salomé... En tout cas, au delà de ces syncrétismes mythologiques assez fantaisistes, ce qui compte le plus dans ce poème est l'attitude mélancolique de la reine, qui pressent douloureusement la fin de son appartenance au monde des humains, selon les traits du personnage médiéval, ainsi que son inquiétante métamorphose : non celle qu'elle devait subir tous les samedis, en devenant «serpente du nombril en aval », mais la métamorphose finale, «la métamorphose animale [...] cette fois complète : elle se mue en une serpente grant et grosse et longue de la longueur de .XV. piez' ${ }^{22}$. En effet, si dans La Forêt bleue Mélusine 
est évoquée comme la reine du château et la fondatrice d'un noble lignage, dans L'Ombre ardente c'est la fée, la dame de la forêt qui est représentée :

Depuis cent ans qu'elle erre au pied des arbres fées,

Elle est fée elle-même, un charme étrange et doux

La fait suivre à minuit des renards et des loups.

Ses yeux au ciel nocturne enchantent les hiboux,

Et près d'elle, érigeant ses fleurs en clairs trophées,

Jaillit un glaïeul rose à feuillage de houx. ${ }^{23}$

En effet, ce sont surtout les fées qui fascinent Jean Lorrain: figures mythiques authentiquement médiévales ( les fées sont nées au XII ${ }^{e}$ siècle $»^{24}$ ), elles peuvent revêtir deux rôles différents, celui de la fée marraine ou celui de l'amante surnaturelle, sans jamais vraiment se départir d'un certain lien avec l'univers satanique, comme le prouvent les "chevauchées nocturnes des "bonnes dames" du Roman de la Rose, conduites par Dame Abonde, Diane ou Hérodiade $»^{25}$ : cette transformation démoniaque de trois figures mythiques de l'antiquité ne pouvait manquer d'attirer l'attention de Jean Lorrain, qui leur consacre les trois poèmes d'ouverture de la section « Le Pays des Fées » de la Forêt Bleue, republiée dans L'Ombre ardente ; ainsi,

Diane a déserté les bois de Cythéron

Et sur sa trace ont fui les nymphes et l'émeute

Des trompes et des cerfs éventrés par la meute...

Elle a cédé l'Hellade au nain vert d'Oberon ;

Et les monts de Judée et le torrent Cédron

Voient, les vendredis saints, hurlante, échevelée

Passer la meute en feu de la grande exilée. ${ }^{26}$

De même,

Aux sons des cors vengeurs la chasse errante et blême

S'enfonce à l'horizon des vieux monts de Bohème

[...]

Quand tinte au clair de lune une cloche de fer

Fêlée, en rugissant la meute vagabonde

Détale, un vent maudit l'emporte et dame Habonde,

Cadavre au ciel errant, remonte et fuit dans l'air,

Emportant dans la trombe et le regret amer

Du passé, le cortège aujourd'hui satanique

Et jadis adoré de la déesse Unique. ${ }^{27}$

Et voici Hérodiade, transformée en une furieuse sorcière démoniaque :

Au fauve appel des cors, au bruit rageur des cistres

La grande Hérodiade et ses nymphes sinistres

Sur des balais fourbus chevauchent en plein ciel.

Des démons accrochés aux crins de leurs cavales,

Elles vont, ventre à terre, au-dessus d'Israël

Et la haine implacable, éclair froid et cruel,

Luit dans leurs grands yeux morts, emplis de larmes pâles.

Entre leurs poings crispés serrant leurs fronts muets,

Sous les grands ciels de cuivre et les lunes brumeuses,

Au dessus des détroits et des villes fumeuses,

Elles vont emplissant l'air de grands coups de fouets ;

Et dans des cors d'airain des nains aux bras fluets,

De Sicile en Brabant, de Mayence à Grenade,

Clament : «Chrétiens, voici la chasse Hérodiade ». ${ }^{28}$ 
Mais les chevauchées infernales sont désormais perdues, vaincues par la religion, qui leur substitue le merveilleux chrétien, avec quelques-unes de ses figures mythiques médiévales :

Le bois moderne est bon chrétien.

[...] La chasse errante sous la lune

De Diane et du roi païen

S'est perdue au loin sur la dune

Aux sons du cor de Saint Julien

Heureux si dans cette déroute,

Qui fait hélas ! le bois désert,

Il nous reste au bord de la route

Le grand cerf blanc de Saint Hubert. ${ }^{29}$

Toutefois les saints figurent mal dans les cordes de Jean Lorrain, qui continue de leur préférer les fées, surtout quand elles sont belles, mystérieuses et fatales; les portraits d'Oriane, Uelda, Tiphaine, Urgèle, Voguelinde, Flosshilde défilent ainsi sous les yeux éblouis du lecteur, parées de simarres, de hennins, de bijoux, de bagues et de fleurs : les fées charmantes " aux yeux verts d'onde, aux yeux verts de couleuvres » ${ }^{30}$, évoluent, dansent ou songent immobiles et impénétrables dans des paysages sauvages et féeriques.

Mais il y a entre toutes une légende qui est pour Lorrain une véritable "obsession", celle de Brocéliande, et une fée à laquelle il a voué une " espèce d'amour posthume " ${ }^{31}$ : Viviane. Comme le rappelle Jean de Palacio dans sa préface à Sonyeuse, « lorsque Jean Lorrain publie Sonyeuse, [...] Brocéliande [...] n'est nullement rélégué au rang de mythe périmé et poussiéreux. Lorrain lui-même affirmait dès 1886 : "La forêt de Brocéliande est le grand enchantement des contes du Moyen Âge" $»^{32}$. À Viviane, à Merlin, à Brocéliande, Lorrain consacre trois différents sonnets, chacun ayant pour titre "Viviane ", dans trois de ses recueils : Le Sang des dieux (1882), La Forêt bleue (1883), L'Ombre ardente (1897) ; puis un long poème de 314 vers, «Brocéliande », publié dans La Forêt bleue et repris dans L'Ombre ardente, sans compter Viviane. Conte en un acte (1885) et Brocéliande (1906), écrits pour le théâtre, et plus particulièrement pour Sarah Bernhard, qui jamais ne les joua, et enfin Sonyeuse (1891), la nouvelle «construite sur cette irruption de la vie moderne dans un passé figé, alliant les conditions d'un conte féerique intemporel [...] et d'une chronique contemporaine ${ }^{33}$.

En délaissant complètement l'identification de Viviane à la Dame du Lac, et donc son rôle bienfaisant de mère nourricière, Jean Lorrain, selon d'ailleurs la leçon des textes médiévaux plus anciens, ne garde que son rôle maléfique de "perfide amie ${ }^{34}$ de Merlin. Il met en scène « le thème du sage trompé par une femme " ${ }^{35}$, bien médiéval luiaussi, revu et corrigé certes selon l'optique et l'esthétique décadente.

Viviane constitue un modèle bien accompli de la femme fatale : elle est «courtisane $~^{36}$, elle est "lascive fée $»^{37}$, "fée experte et savante $»^{38}$, elle est, aux dires de Merlin, « esprit froid et rusé, douce et perfide dame, dame périlleuse $»^{39}$. Sa beauté est sans pareil : son immense et lourde chevelure «d'or fauve $»^{40}$, «d'or lisse et fluide $»^{41}$, peut l'envelopper tout entière et envelopper le mage, pour le séduire :

La dame, entre ses doigts faisant couler ses tresses,

Les ouvrit et, roulant en soyeuses caresses

Leur or lisse et fluide oint d'essence et de nard

Sur la barbe neigeuse et le cou du vieillard,

« M'aimez-vous, ô Myrdhinn, m'aimez-vous puissant maittre »

Implora-t-elle $[. . .]^{42}$

Cahiers de recherches médiévales, 11 | 2004 
yeux, " enivrants comme un philtre $»^{43}$, sont couleur de turquoise; sa figure est «blanche, attirante et souple $»^{44}$, « svelte » et volontiers $~ «$ demi-nue $»^{45}$; ses doigts sont «blancs et légers $»^{46}$, ses bras, « errants et frais $»^{47}$; ses pieds sont " diaphanes $»^{48}$, son rire est "ailé $»^{49}$, « jeune et frais $»^{50}$, «argentin $»^{51}$; au moment de son triomphe, sa beauté resplendit, superbe et cruelle :

Et férocement rousse et férocement nue, Les seins droits et pourprés, rouge tentation, Le heaume de Myrdhinn sur l'or en fusion De ses fauves cheveux bondissant sur ses hanches,

Viviane apparut, farouche, entre les branches. ${ }^{52}$

Mais Viviane-femme fatale de la fin de siècle, ne peut pas se contenter de ses qualités naturelles; parfums, vêtements, bijoux parent ses attraits, en offrant un exemple délicieux de femme artificielle: myrrhe, cervoise, nard, "parfums troublants " ${ }^{33}$ embaument tout le corps et les cheveux de Viviane ; «sa robe aux longs plis " $^{54}$ est une tunique «fleurie sur un fond vert de mer d'arabesques d'argent $»^{55}$; sur ses lourdes tresses elle porte « un hennin brodé » et « doré $»^{56}$ ou bien « un étroit tortil d'or » ${ }^{57}$; ses bras nus sont " chargés d'anneaux d'orfroi $»^{58}$ qui vont « des poignets aux aisselles $»^{59}$; souvent, pour compléter sa gracieuse figure modern style, Lorrain la pare d'une décoration florale, comme dans le tableau qu'il nous offre d'elle et de son triomphe dans L'Ombre ardente :

Aussi pour mieux marquer sa gloire et son dédain,

Sur sa crinière d'or elle a du vieux Myrdhinn,

Mage et preux, arboré la couronne et le casque.

Le casque a pour cimier un mufle de tarasque,

La Dame a pour défi son mépris souverain

Et sous son rouge orteil jaillit un lys fantasque..$^{60}$

Enfin, Viviane est très souvent représentée pendant la danse magique qui enchantera Merlin pour toujours, « où la danse [...] apparaît bien - écrit Jean de Palacio - comme une manœuvre de séduction et l'exercice de la puissance du corps ${ }^{61}$, mais aussi comme une « assimilation implicite de Viviane à Salomé » ${ }^{62}$.

Ainsi, le personnage de la fée Viviane, «figure obsessionnelle dans la production de l'auteur, [...] une autre figuration de la Grande Luxure $»^{63}$, apparait bien dans les vers du poète comme l'éblouissante résurgence d'un mythe médiéval (enjolivé par le goût modern style) que Lorrain cependant transforme d'une manière radicale par ce que Jean de Palacio définit comme "merveilleux perverti ${ }^{64}$; le poète opère en effet un dévoiement des rôles des deux personnages : si dans la littérature médiévale "Viviane, la fée sylvestre, [...] se refuse à Merlin par refus de l'amour " ${ }^{65}$ dans «la hantise de préserver sa virginité » ${ }^{66}$, et la terrible fin de Merlin (" fils du démon [à qui] s'attache le péché de la luxure $\left.»^{67}\right)$, « a la signification d'un châtiment $»^{68}$, dans les vers de Lorrain c'est Viviane, l'enivrante femme fatale, qui s'offre en exhibant sans pudeur les arts les plus raffinés de la séduction luxurieuse, pour parvenir à ses fins et provoquer la perte de Merlin l'enchanteur. Celui-ci au contraire, "mage et preux »" est "le myre indulgent $»^{70}$, il est l'homme savant, très sage et très réservé, très clairvoyant aussi : il est capable de deviner toutes les manœuvres de la femme, qu'il aime en effet, d'une manière bien discrète, qu'il admire beaucoup et envers qui il donne preuve d'une indulgente tendresse, à laquelle il oppose cependant un refus très courtois mais bien ferme, en décidant enfin de mettre un terme aux embûches périlleuses de la perfide dame en essayant de l'endormir pour toujours; mais voilà, "Myrdhinn, vieux loup 
royal au piège enfin surpris ${\aleph^{71}}$, sera vaincu par l'impitoyable Viviane, jeune et forte, qui s'empare des moyens magiques de l'enchanteur, en se débarrassant de lui dès qu'elle a eu ce qu'elle voulait et en riant cruellement de ses larmes et de sa défaite.

En réalité, Merlin apparaît désormais comme un vieillard au "front chauve », à la «barbe argentée ${ }^{72}$; peut-être aurait-il aimé céder aux séductions de la femme : il en est « ébloui, les yeux extasiés ${ }^{73}$; mais il n'en a plus les moyens et il dénonce lui-même, dans une douloureuse complainte, "l'usure du temps», "les ravages de la décrépitude $»^{74}$ :

Je suis trop vieux pour vous belle dame amoureuse,

Trop las pour votre ardeur attirante et fiévreuse,

Trop usé pour le rêve et l'impossible essor,

Que médite votre âme ; et, parmi vos crins d'or,

En vain, voluptueuse et morbide couleuvre,

Pour me plaire avez-vous, enfant, mis tout en œuvre ;

Le sang de mes vingt ans, que l'âge a refroidi,

Trahit le vieux Myrdhinn et, dans l'ombre engourdi,

Songeur lourd de regrets, de tristesse et d'années,

Je suis le morne époux des vieilles fleurs fanées. ${ }^{75}$

C'est dans cette résignation amère et sans espoir que nous reconnaissons le chiffre bien personnel apposé par Jean Lorrain à la matière de Bretagne.

On pourrait se demander combien a compté l'œuvre wagnérienne dans l'attraction de Jean Lorrain pour Brocéliande et ses personnages merveilleux. Très sensible à toutes les modes, il les a certainement suivies aussi dans ce domaine. Il ne faut pas toutefois sousestimer la grande passion de son enfance pour les contes de fées et pour les légendes ; il écrit dans Sonyeuse à propos d'un grand parc abandonné :

Je n'avais que douze ans, mais, liseur enragé de romans de chevalerie et le cerveau déjà farci de récits épiques et d'histoires fabuleuses, j'avais, dans mon imagination d'enfant, baptisé ce coin feuillu et solitaire du nom charmant de Brocéliande.

Brocéliande, la forêt des pommiers du pays de Bretagne, où l'astucieuse et svelte fée Viviane prit à son piège le vieux mage Myrdhinn. ${ }^{76}$

Ce sont encore la fée et le sorcier qui ressortent de ces pages, tandis qu'on chercherait en vain dans l'œuvre de Lorrain Lancelot ou Parsifal ; le roi Arthur est à peine nommé ; jamais on ne parle du Graal. D'ailleurs, s'il est vrai que l'influence de Wagner a été immense, il faut reconnaître, comme l'écrit Isabelle Cani, qu'« en France, le Graal est alors [au tournant des $\mathrm{XIX}^{\mathrm{e}}-\mathrm{XX}^{\mathrm{e}}$ siècles] une référence culturelle, non un mythe littéraire [...]. Dans la matière de Bretagne, il est des parts plus françaises : Lancelot du Lac, l'inquiétante fée Morgane [...], l'histoire de Merlin et de Viviane qui est de toutes la moins oubliée $\aleph^{77}$.

Le commentaire qu'Isabelle Cani écrit dans son article sur "Graal et décadence ", semble s'appliquer parfaitement aux poèmes de Jean Lorrain :

Parti à la recherche du Graal, on se retrouve devant son opposé. Au lieu de l'élévation, l'enfouissement. Au lieu de la robe d'or et du sang lumineux, les couleurs froides de l'eau dormante. Au lieu du chevalier pur, la femme fatale. Au lieu de la virginité nécessaire, l'envoûtement sexuel. Au lieu du sauveur qui délivre, le charme qui emprisonne. ${ }^{78}$

En effet, dans l'œuvre de Jean Lorrain la quête est totalement absente, ce qui autorise Jean de Palacio à souligner que paradoxalement les contes merveilleux des Princesses d'ivoire et d'ivresse relèvent « plus du naturalisme que de la féerie, sorte, si l'on veut, de naturalisme baudelairien $»^{79}$. 

avant tout le désir d'y puiser le portrait le plus fascinant de la femme fatale, qu'il s'efforce de construire à la manière d'un tableau. Cette passion pour la peinture, ce goût ekphrastique qui sont présents dans toute l'œuvre de Jean Lorrain, e non seulement dans son œuvre poétique, constituent - on le sait bien - l'une des techniques les plus significatives des auteurs parnassiens. En effet, comme l'écrit Philippe Jullian, «Lorrain [...] restait parnassien par la forme même de ses poèmes, plus proche de Heredia que de Mallarmé $»^{80}$. Cependant le même critique reconnaît que les peintres qu'inspirent Lorrain ne sont certes pas ceux qu'aimaient les auteurs du parnasse, mais ceux qui étaient tant à la mode chez les symbolistes : Gustave Moreau, Odilon Redon, les préraphaélites, Beardsley, Jeanne Jacquemin ; et le poème de Brocéliande que nous avons tant de fois cité ne serait lui-aussi rien d'autre que la reproduction d'un tableau :

Tout un long poème relatant l'enchantement de Merlin, appelé Myrdhinn par la fée

Viviane, est comme la glose d'un tableau de Burnes-Jones peint en 1875:

L'Enchanteur vieilli, immobilisé dans le tronc d'un arbre en fleurs. ${ }^{81}$

Ainsi donc Jean Lorrain, par le choix de ses modèles préférés, s'avère une fois de plus comme l'un des décadents de la plus stricte obédience : le Moyen Âge dont il raffole, et que peut-être il connaît bien, n'est lu qu'à travers l'écran déformant d'une peinture moderne et volontiers arbitraire; ce qui plus est, le fait d'ordonner souvent ces portraits en séquences analogues, la prolifération des objets, des vêtements, des bijoux, des décorations florales qui parent et encadrent ces princesses angéliques et insensibles, ces reines hautaines et inquiétantes, ces fées cruelles et enchanteresses, constituent le chiffre exquis du maniérisme de Lorrain, en même temps que sa volonté de distanciation : ce «triste et fol amant d'anciens portraits $»^{82}$ peut contempler une femme fatale sans le danger de tomber dans le piège de ses charmes, si elle n'est qu'un tableau splendide, enfermé dans un cadre précieux.

La deuxième raison qui pousse Jean Lorrain, « cœur épris des temps légendaires $»^{83}$, à évoquer quelques mythes du Moyen Âge est sa grande passion pour le merveilleux :

Il n'est pas au monde émotion un peu délicate - écrit-il - qui ne repose sur l'amour du merveilleux : l'âme d'un paysage est tout entière dans la mémoire, plus ou moins peuplée de souvenirs, du voyageur qui le traverse, et il n'y a ni montagnes, ni forêts, ni levers d'aube sur les glaciers, ni crépuscules sur les étangs pour qui ne désire et ne redoute à la fois voir surgir Oriane à la lisière du bois, Tiphaine au milieu des genêts et Mélusine à la fontaine. ${ }^{84}$

Ce sont les contes de fées, ce sont les anciennes légendes, c'est son amour pour le merveilleux qui s'offrent à Lorrain comme un remède contre le naturalisme et lui consentent de mettre entre parenthèses la réalité « du Paris frelaté de 1900, où [il] situe la plupart de ses histoires $»^{85}$. Mais la pression de la réalité ne cesse jamais de s'exercer sur cet auteur, en pénétrant, contre son gré, même dans ses rêveries les plus antinaturelles, mythiques et féeriques; ainsi elle donne lieu à ce que nous avons accepté de définir (selon l'indication de Jean de Palacio) comme le «merveilleux perverti » qui marque toute l'œuvre de Lorrain. Ainsi que le souligne cet éminent spécialiste de la fin de siècle en commentant les contes des Princesses (mais cette analyse est également valable pour les poèmes), "une sourde inquiétude ${ }^{86}$ surgit constamment du merveilleux :

Le stéréotype rassurant du conte de fées traditionnel est souvent battu en brèche.

[...] C'est que les contes merveilleux de Lorrain sont aussi des contes cruels. Les

blessures y saignent et les têtes y tombent. [...] Une poétique de la putrescence et de 
la charogne s'y installe, instaurant une forme encore plus subtile de cruauté qui se plaît à décrire les ravages de la mutilation ou de la décrépitude. ${ }^{87}$

Ainsi, la Belle au bois dormant préfère continuer son sommeil pour toujours " plutôt que vivre et que souffrir » :

Elle a mieux aimé, la charmante,

Reprendre son rêve enivrant

Que vivre en notre âpre tourmente. ${ }^{88}$

41 Ainsi, le poète contemple avec désolation le passé, qui est comme un étang mort où son âme se corrompt, où l'on cherche vainement à faire revivre le merveilleux :

Un trou saignant au front, les Espérances fées

De longs glaieuls flétris et de lys morts coiffées,

Au son charmeur du cor ne s'éveilleront plus. ${ }^{89}$

Ainsi, le prince charmant, après de longues recherches, trouve enfin la belle fileuse, qui "se mine d'amour en [l'] attendant ", enfermée " dans la vieille tour en ogive » : mais "ô désespoir! Fille et donjon sont en ruine. La belle a l'âge du manoir» et le pauvre prince ne pourra jamais plus se libérer des lierres et des ronces où il restera emprisonné sa vie entière ${ }^{90}$.

Il nous faut reconnaître, pour conclure, que les mythes du Moyen Âge fascinent incontestablement Jean Lorrain, surtout à cause du merveilleux féerique, qui est l'un des caractères fondamentaux de la littérature médiévale, si profondément influencée par la tradition populaire. Cependant, dans ses poèmes une perversion du merveilleux s'opère constamment : derrière la féerie mythique, derrière les vêtements splendides, les bijoux précieux, les pierres rares, derrière les gracieuses décorations modern style et les couleurs éclatantes ou délicates, derrière les yeux charmeurs et les chevelures ruisselantes des reines et des fées, d'autres figures s'imposent, épouvantables et sans aucun ornement cette fois, celles de la vieillesse et de la mort: contre tout effort de fuite et de rêve, c'est toujours la hideuse réalité qui finit inéluctablement par triompher.

\section{NOTES}

1.Je pense par exemple à Ph. Jullian, Jean Lorrain ou le satiricon 1900, Paris, Fayard, 1974 ou à M. Praz, La Carne, la morte e il diavolo nella letteratura romantica (1931), Firenze, Sansoni, 1966 ; cet éminent spécialiste consacre à Lorrain plusieurs pages, mais il éprouve le besoin de justifier avec insistance son choix en réservant toujours à cet auteur une lourde ironie. Quant aux études que j'ai moi-même publiées sur J. Lorrain, je me permets de renvoyer à «Il Narciso speculare di Jean Lorrain », Narciso allo specchio : dal mito al complesso, dir. E. Mosele, Schena, Fasano, 1995, pp. 127-141, et à « Le fiabe di Jean Lorrain o il marchingegno trionfante ", Antimimesis, dir. G. Bogliolo et P. Toffano, Fasano, Schena, 1997, pp. 161-187.

2.Ph. Jullian, op. cit., p. 60.

3.Thibaut d'Anthonay, Jean Lorrain, barbare et esthète, Paris, Plon, 1991, pp. 29-30 et 48. 
4.G. Ducrey, Introduction à J. Lorrain, Monsieur de Bougrelon, Romans fin-de-siècle, Paris, Laffont, p. 89.

5.Ph. Jullian, op. cit., p. 165.

6.Ibid., p. 12.

7.A.M. Finoli, « Immagini del Medio Evo nei romanzi popolari francesi dell'Ottocento » (1983), Prose di Romanzi, raccolta di studi (1979-2000), Milano, Led, 2001, p. 28.

8.Ibid., p. 30.

9.Ibid., p. 28.

10.J. Lorrain, « Un peu de musique », La Forêt bleue, Paris, Alphonse Lemerre, 1883, vv. $1-4$, p. 40.

11.J. Lorrain, « Il passe un bohémien », La Forêt bleue, éd. cit., vv. 1-4, 9-11, p. 95.

12.J. Lorrain, « Le Voyageur » IV, Les Griseries, Paris, Tresse \& Stock, 1887, vv. 1-8, p. 49.

13.J. Lorrain, Princesses d'ivoire et d'ivresse, Paris, Séguier, 1993, pp. 24-26.

14.J. Lorrain, « Récurrence », Les Griseries, éd. cit., v. 12, p. 90 et L'Ombre ardente, Paris, Charpentier et Fasquelle, 1897, p. 12.

15.J. Lorrain, « Vieux conte », I, Les Griseries, éd. cit., pp. 121-122.

16.J. Lorrain, « Vieux conte », II, vv. 12-15, pp. 123-124.

17.J. Lorrain, « Elaine », L'Ombre ardente, éd. cit., vv. 1-4 et 9-14, p. 191.

18.J. Lorrain, « Les Héroïnes », L'Ombre ardente, éd. cit., vv. 21-40 et 45-56, pp. 188-189.

19.J. Lorrain, « Mélusine », La Forêt bleue, éd. cit., p. 18.

20.L. Harf-Lancner, Les Fées au Moyen Âge, Paris, Champion, 1984, p. 170.

21.Jean d'Arras, Mélusine, éd. L. Stouff (1932), Genève, Slatkine, 1974, p. 253.

22.L. Harf-Lancner, op. cit., p. 175.

23.J. Lorrain, « Mélusine », L'Ombre ardente, éd. cit., vv. 9-14, p. 193.

24.L. Harf-Lancner, op. cit., p. 77.

25.Ibid., p. 30.

26.J. Lorrain, « Diane », La Forêt Bleue, éd. cit., vv. 8-14, p. 12 et L'Ombre ardente, éd. cit., p. 66.

27.J. Lorrain, « Dame Habonde », La Forêt Bleue, éd. cit., vv. 1-2, 8-14, p. 14 et L'Ombre ardente, éd. cit., p. 68.

28.J. Lorrain, « Hérodiade ", La Forêt Bleue, éd. cit., p. 13 et L'Ombre ardente, éd. cit., p. 67. 29.J. Lorrain, "Clair de Lune ", La Forêt Bleue, éd. cit., vv. 20, 25-32, p. 54 et L'Ombre ardente, éd. cit., p. 75.

30.J. Lorrain, «Urgèle », La Forêt Bleue, éd. cit., v. 4, p. 19 et L'Ombre ardente, éd. cit., p. 180.

31.J. Lorrain, Sonyeuse, Paris, Séguier, 1993, p. 62.

32.J. de Palacio Préface à J. Lorrain, Sonyeuse, éd. cit., p. 7. Les paroles de Lorrain sont tirées d'une lettre à François Coppée, octobre 1886, inédite, coll. part. (cf. ibid., n. 2, p. 7).

33.J. de Palacio, Préface à J. Lorrain, Sonyeuse, éd. cit., pp. 9-10.

34.L. Harf-Lancner, op. cit., p. 191.

35.Ibid.

36.J. Lorrain, « Viviane », Le Sang des dieux, Paris, Lemerre, 1882, vv. 10 et 12, p. 27.

37.J. Lorrain, « Viviane », La Forêt bleue, éd. cit., v. 15, p. 27.

38.J. Lorrain, "Brocéliande », La Forêt bleue, éd. cit., v. 28, p. 82 et L'Ombre ardente, éd. cit., p. 110.

39.J. Lorrain, « Brocéliande », La Forêt bleue, éd. cit., vv. 180, 222, 225 pp. 88-89 et L'Ombre ardente, éd. cit., pp. 116-119. 
40.J. Lorrain, « Brocéliande », La Forêt bleue, éd. cit., v. 17, p. 81 et L'Ombre ardente, éd. cit., p. 109.

41.J. Lorrain, « Brocéliande », La Forêt bleue, éd. cit., vv. 31, p. 82 et L'Ombre ardente, éd. cit., p. 110.

42.J. Lorrain, « Brocéliande », La Forêt bleue, éd. cit., vv. 29-34, p. 82 et L'Ombre ardente, éd. cit., p. 110.

43.J. Lorrain, « Viviane », L'Ombre ardente, éd. cit., v. 4, p. 192.

44.J. Lorrain, «Viviane ", Le Sang des dieux, éd. cit., v. 3, p. 27.

45.J. Lorrain, « Viviane », La Forêt bleue, éd. cit., v. 9, p. 15 et L'Ombre ardente, éd. cit., p. 69.

46.J. Lorrain, « Brocéliande ", La Forêt bleue, éd. cit., v. 231, p. 90 et L'Ombre ardente, éd. cit., p. 119.

47.J. Lorrain, «Brocéliande », La Forêt bleue, éd. cit., v. 232, p. 90 et L'Ombre ardente, éd. cit., p. 119.

48.J. Lorrain, « Viviane ", Le Sang des dieux, éd. cit., v. 7, p. 27.

49.J. Lorrain, «Brocéliande », La Forêt bleue, éd. cit., v. 285, p. 92 et L'Ombre ardente, éd. cit., p. 122.

50.J. Lorrain, « Brocéliande », La Forêt bleue, éd. cit., v. 269, p. 91 et L'Ombre ardente, éd. cit., p. 121.

51.J. Lorrain, « Viviane », La Forêt bleue, éd. cit., v. 6, p. 15 et L'Ombre ardente, éd. cit., p. 69.

52.J. Lorrain, « Brocéliande », La Forêt bleue, éd. cit., vv. 286-290, p. 92 et L'Ombre ardente, éd. cit., p. 122.

53.J. Lorrain, « Viviane ", Le Sang des dieux, éd. cit., v. 4, p. 27.

54.J. Lorrain, « Brocéliande », La Forêt bleue, éd. cit., v. 243, p. 90 et L'Ombre ardente, éd. cit., p. 120.

55.J. Lorrain, « Brocéliande », La Forêt bleue, éd. cit., vv. 10-11, p. 81 et L'Ombre ardente, éd. éd. cit., p. 109.

56.J. Lorrain, « Brocéliande ", La Forêt bleue, éd. cit., v. 9, p. 81, v. 295, p. 92 et L'Ombre ardente, éd. cit., pp. 109, 122.

57.J. Lorrain, « Viviane », Le Sang des dieux, éd. cit., v. 4, p. 27.

58.J. Lorrain, « Brocéliande », La Forêt bleue, éd. cit., v. 15, p. 81 et L'Ombre ardente, éd. cit., p. 109.

59.J. Lorrain, « Brocéliande », La Forêt bleue, éd. cit., v. 185, p. 88 et L'Ombre ardente, éd. cit., p. 116.

60.J. Lorrain, « Viviane ", L'Ombre ardente, éd. cit., vv. 9-14, p. 192.

61.J. de Palacio, Préface à Jean Lorrain, Sonyeuse, éd. cit., p. 15.

62.Ibid.

63.J. de Palacio, Figures et formes de la décadence, Paris, Séguier, 1994, pp. 49-50.

64.J. de Palacio, Présentation à J. Lorrain, Princesses d'ivoire et d'ivresse, éd. cit., p. 7.

65.L. Harf-Lancner, op. cit., p. 292.

66.Ibid., p. 294.

67.Ibid., p. 298.

68.Ibid., p. 296.

69.J. Lorrain, « Viviane », L'Ombre ardente, éd. cit., v. 11, p. 192.

70.J. Lorrain, « Brocéliande », La Forêt bleue, éd. cit., v. 127, p. 86 et L'Ombre ardente, éd. cit., p. 114. 
71.J. Lorrain, « Brocéliande », La Forêt bleue, éd. cit., v. 280, p. 92 et L'Ombre ardente, éd. cit., p. 122.

72.J. Lorrain, « Brocéliande », La Forêt bleue, éd. cit., vv. 18-19, p. 81 et L'Ombre ardente, éd. cit., p. 109.

73.J. Lorrain, « Brocéliande », La Forêt bleue, éd. cit., v. 76, p. 83 et L'Ombre ardente, éd. cit., p. 112.

74.J. de Palacio, Présentation à J. Lorrain, Princesses d'ivoire et d'ivresse, éd. cit., pp. 22 et 12.

75.J. Lorrain, « Brocéliande », La Forêt bleue, éd. cit., vv. 129-138, p. 86 et L'Ombre ardente, éd. cit., pp. 114-115.

76.J. Lorrain, Sonyeuse, éd. cit., p. 61.

77.I. Cani, «Graal et décadence : l'avortement d'un mythe », dir. A. Montandon, Mythes de la décadence, Presses Universitaires Blaise Pascal, Clermont-Ferrand, 2001, pp. 205 et 212.

78.Ibid., p. 212.

79.J. de Palacio, Présentation à J. Lorrain, Princesses d'ivoire et d'ivresse, éd. cit., p. 13. 80.Ph. Jullian, op. cit., p. 98.

81.Ibid., p. 35,

82.J. Lorrain, « Récurrence », in L'Ombre ardente, éd. cit., v. 14, p. 12.

83.J. Lorrain, « Résurrection », in L'Ombre ardente, éd. cit., v. 2, p. 9.

84.J. Lorrain, Princesses d'ivoire et d'ivresse, éd. cit., p. 24.

85.G. Ducrey, op. cit., p. 90.

86.J. de Palacio, Présentation à J. Lorrain, Princesses d'ivoire et d'ivresse, éd. cit., p. 11.

87.Ibid., pp. 11-12.

88.J. Lorrain, « La Belle au bois qu'a réveillée ", L'Ombre ardente, éd. cit., vv. 10-12, p. 88.

89.J. Lorrain, « L'Étang mort », L'Ombre ardente, éd. cit., vv. 12-14, p. 63.

90.J. Lorrain, « Prologue », La Forêt bleue, éd. cit., vv. 59-60, 62, 82-84, pp. 33-34.

\section{AUTEUR}

LIANA NISSIM

Università degli Studi, Milan 